\title{
Über den Werth des biologischen Experiments.
}

Von

\author{
Hans Driesch.
}

Eingegangen am 5. Februar 1897.

Wer meine Schriften kennt, weiß, dass ich mit sehr vielen der Erörterungen, welche im zweiten Heft von Oscar Her'TwiG's "Zeitund Streitfragen der Biologie enthalten sind, durchaus einverstanden bin. Er weiß, dass ich z. B. den gegen die Specialarbeiten Roux's gerichteten »Anhang « (pag. 98 bis Ende) im Großen und Ganzen unterschrciben würde, dass auch ich das Wort »Entwickelungsmechanik" beanstande. Wende ich dieses Wort doch schon seit geraumer Zeit nicht mehr an, sondern gebrauche, um das Studium der Entwickelung, des Sich-Entwickelns um seiner selbst willen, nicht zum Zwecke der Vergleichung, za bezeichnen, die Worte Entwickelungsphysiologie oder Entwickelungsanalytik, ebenso wie man von einer Verdauungsphysiologie, einer Nervenphysiologie, aber nicht von einer Verdauungsmechanik, einer Nervenmechanik zu reden pflegt. Demn es ist außer Zweifel, dass das Wort Mechanik hier zu viel präjudicirt, wenn es eng gefasst wird, und dass sein Inhalt sich rerfliussigt und nichts besagt, wenn man ihn weit fasst: „der Causalität unterstehendes Geschehen" (Roux) nämlich würden die Lebensphänomene auch damn sein, wenn es sich als nöthig erweisen sollte, an Stelle der "Maschinentheorie des Lebens \& 1 ) eine Theorie des Vitalismus za begründen. Solche, die in diesem Falle von Metaphysik reden wiurden, würden dadurch kund thun, dass sie die Bedeutung der Worte Causalität und Metaphysik nicht klar erfasst haben.

Herrsclit somit in vielen Dingen zwischen Oscar Hertwig nud mir eine erfrenliche Harmonie der wissenschaftlichen Ansichten, so

1) Biol. Centralbl. XVI. 
ist das doch in einem Punkte nicht der Fall, nämlich in der Werthschätzung des Experiments, und hier meine Abweichung der Meinung auszusprechen, kann ich um so weniger unterlassen, als mir die Gefahr vorzuliegen scheint, dass eine gewisse nicht kleine Gruppe von Biologen durch die Heriwig'sehen Ausführungen in einem sehr unberechtigten Selbstgefühl bestärkt werden und in ihnen gleichsam einen Freibrief für die eigne Urtheilslosigkeit erblicken wird.

Gegen Solche also, die sich mit HerTwig's Angriffen werden decken wollen, schreibe ich hier, weniger gegen Hertwig selbst; denn er hat, trotz seiner Angriffe auf den hohen Werth des Versuchs, durch die That zu wiederholten Malen gezeigt, dass er das Experiment zu schätzen versteht - vielleicht sogar mehr, als er in seiner polemischen Schrift zugiebt.

Un die Hertwig'schen Angriffe gegen das Experiment als ein Mittel zur Erforschung der Lebensvorgänge, das die einfache Beobachtung an Rang überragt, zu entkräften, müssen wir zunächst nach einer klaren Definition des Begriffs des Experiments suchen oder, noch besser, erst betrachten, welche Definition dieses Begriffs HER'TwiG aufstellt.

Da erfahren wir denn auf pag. 65 seiner Schrift, dass das Experiment seine eigentliche Bedeutung im Anorganischen habe: die anorganischen Körper seien »verhältnismäßig unveränderlich «, durch das Experiment zwänge der Mensch »die Stoffe, sich zu verändern« und mache sie dadurch erst der Forschung zugänglich; denn es sei "klar, dass nur Dinge, so weit sie sich verändern, Gegenstand causaler Erkenntnis sein könuen

Sehen wir einmal davon ab, dass es doch recht bedenklich ist, zu sagen, die unorganischen Körper seien *verhältnismäßig unveränderlich " (das Erleben eines tuichtigen Regens oder Sturmes kann diese Aussage in einfachster Weise widerlegen): also ein Mittel um Veränderungen herbeizuführen ist nach HerTwigr der Versuch.

Wir halten dafür, dass diese Definition gerade das Wesentliche am Begriff des Versuchs nicht trifft: das Experiment will nicht allgemein Veränderungen herbeiführen, sondern will Veränderungen (Vorgänge) nach Belieben isoliren oder variiren und isolirte Vorgänge bewusst kombiniren.

Nicht weil die anorganische Natur »verhältnismäßig unveränderlich « ist, haben der Physiker und der Chemiker den Versuch erfunden, sondern weil sie sich in so ungeheuer mannigfacher und verwobener Art, wenn auch oft langsam, verändert, dass aus Beobach- 
tung des Gegebenen nie und nimmer Causalzusammenhänge mit Sicherheit ermittelt werden können. Erst dadurch, dass Vorgänge $A, B, C$ etc. bewusst zu bestimmter Zeit und an bestimmtem Ort isolirt hervorgerufen sind, ist man in der Lage anzugeben, welche Vorgänge $A^{\prime}, B^{\prime}, C^{\prime}$ ete. im Causalverhältnis mit ihnen stehen.

Ganz dasselbe, was den Werth des Versuchs in den anorganischen Disciplinen ausmacht, trifft nun auch vom biologischen Versuch zn. Bevor wir aber daran gehen, den Werth des Experimentalverfahrens in der Biologie und speciell in der Entwickelungsphysiologie zu erforschen, haben wir uns mit Herswig über die Begriffe $»$ Kraft « und »Causalität aus einander zu setzen. Es wird sich nämlich zeigen, dass sein Urtheil abber das Experiment ganz wesentlich von der Art und Weise abhängt, in der er diese Begriffe, zumal den der Causalität, praktisch anwendet.

Im Anschluss an Lotze und Andere eifert HerTwig dagegen, Körpern, von denen eine Wirkung ausgelit, $d$. $h$. an denen eine Veränderung eine andere Veränderung an anderen Körpern zur Folge hat, eine wirkende $\gg$ Kraft « beizulegen; es sei, so sagt er, damit nur eine Scheineinsicht gewonnen; klarer und sachentsprechender sei es, einfach zu sagen: hier geht ein abhängiges Veränderungsgeschehen nach dieser oder jener Regel, d. h. eine bestimmte Art causalen Geschehens vor sich. Wer, nach dem Vorbild des ARIsToteles ${ }^{1}$ ), dem sich verändemden Körper $A$ cine "Veränderungsfähigkeit «, eine

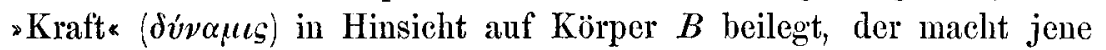
ein Verhältnis betreffende Regel, jene »Wirkungsweise \&, zu einer Eigenschaft und glaubt fälschlich, damit mehr als ein neues Wort gewounen zu haben.

Wir wollen dieses in letzter Zeit oft ${ }^{2}$ ) dargelegte Raisonnement hier nicht auf seine Richtigkeit prifen, dieselbe sei vielmehr zum Zwecke des Folgenden als erwiesen vorausgesetzt.

Gut, also wir vermeiden den Begriff der »wirkenden Kraft"; mich dünkt aber, sehr häufig, wenn ein Autor olne nähere Erläuterung von "Kräften" redete, hat er dieses Wort gar nicht im sinne der einem Körper beigelegten $»$ wirkenden Kraft« gethan, sondern im

1) Metaphys. Buch IX. KIRchmanx'sehe Übers. 1871. Bd. II. pag. $28 \mathrm{ff}$.

2) In Dreyer's »Studien zu Methodenlehre und Erkenntniskritik * findet sich z. B. auf pag. $90 \mathrm{ff}$, 109, 146 ganz Ähnliches. Hentwig polemisirt gegen Dreyer auf Grund von dessen älterer theoretischer Schrift, offenbar ohne die neuere zu kennen, die uberhaupt unter Biologen viel zu wenig bekannt geworden zu sein scheint. 
Sinne des alten und in der Verwendung recht bequemen Wortes Naturkraft, das auch von Hertwig (z. B. pag. 45) gutgeheißen wird. In diesem Sinne ist z. B. auch von mir bisweilen von Kräften geredet worden, von Kräften $=$ Naturkräften $=$ Wirkungsweisen $=$ Naturgesetzen = Energiearten, meist freilich, indem einer der letztgenannten Ausdritcke erlïuternd in Klammern beigefügt wurde; in diesem Sinne, dünkt mich, könnte man auch das Wort »Kraft", wo es bei Roux vorkommt, ohne gewaltsame Interpretation verstehen 1). Solche Naturkräfte, solche elementare Naturwirkungsweisen würden Wärme, Elektricität, Schwer»kraft « sein.

Lässt man das Wort Kraft in diesem, wie gesagt, auch von Hertwig theoretisch gebilligten Sinne, also als Naturgesetz, demgemäß ein Geschehen verläuft, zu, so gewinnt man einen bequemen Ausdruck für ein wichtiges Element der Naturerforschung, für ein Element, das bei HerTwig praktisch nicht gentigend in seine Rechte tritt.

Damit gehen wir zu einer Erörterung des Begriffs »Causalität" bei Hertwig iiber, welche uns zur Frage nach der Werthsehätzung des biologischen Experiments wiederum iiberleiten wird:

»Alles, was geschieht (anhebt zu sein), setzt etwas voraus, worauf es nach einer Regel folge«, so lesen wir in der »Kritik der reinen Vernunft«. Dieser Satz involvirt Kan'r's Definition der Ursache und Wirkung, der Causalität.

»Jede Veränderung kann nur eintreten dadurch, dass eine andere, nach einer Regel bestimmte, ihr vorhergegangen ist, durch welche sie aber dann als nothwendig herbeigeführt eintritt: diese Nothwendigkeit ist der Causalnexus. " »Ein Naturgesetz ist die der Natur abgemerkte Regel, nach der sie, unter bestimmten Umständen, sobald diese eintreten, jedes Mal verfährt. " So bei Schopenhauer; Hertwig citirt seine Sätze unter Beistimmung.

Also Abhängigkeitsveränderung nach Regeln ist "Causalität«; Ermittelung der Veränderungsregeln der Natur ist also die Aufgabe der »causalen "Forschung.

Uns scheint, dass Hencwig trotz seiner Citate und dem Beifall, den er ihrem Inhalt zollt, diesen in ihnen liegenden Begriff prak-

1) Wenn man in diesem Sinne die von HerTwıg auf pag. 55 bek:̈mpfte Ausfiihrung Dreyer's versteht, so wüsste ich (abgesehen vielleicht von dem etwas seltsamen Symbiosevergleich) uichts gegen sie einzuwenden. Sie besagt dann: »manche Verïnderungen in Organismen gehen nach anorganischen Naturgesetzen vor sich $\lessdot$. 
tisch nicht gewürdigt hat; er wendet nämlich später wiederholt den Begriff des »Causalen « in einem Sinne an, der gerade sein Wesentliches: die Regel, nach der eine Folge verläuft, außer Acht lässt. Da weiterhin gerade in diesem Mangel seine Angriffe gegen den höheren Werth des Versuchs im Vergleich zur bloßen Beobachtung begriindet sind, mïssen wir hier etwas eingehender werden.

Hertwig meint, wenn man die Entwickelung einer Thierart beobachte, erhalte man eausale Resultate; das voransgehende und das folgende Stadium stiinden hier allemal im Verhältnis von Ursache und Wirkung. Wer diesem Satze beistimmen würde, der würde vielmehr, so dünkt uns, auf wirklich *causale Einsicht Verzicht leisten. Wo bleibt hier die »Regel «, nach der die Veränderungsfolge verläuft, wo das Naturgesetz, demgemäß sie sich abspielt? Man miusste denn geneigt sein, so viel »Naturgesetze« zu stabiliren, wie es Paare auf einander folgender, unterscheidbarer Stadien in der Entwickelung aller einzelnen Lebensformen gäbe; damit würde man den Begriff $*$ Naturgesetz* aber verflussigen. Denn nicht weiter analysirbare einfache oder doch wenigstens auf Einfachheit zustrebende Regeln des Geschehens pflegen die anorganischen Wissenschaften als Naturgesetze zu bezeichnen, nicht aber etwa die Art der Folge komplexer geologischer Zustände.

Wenn wir also wissen, dass in der Entwickelung des Frosches der "Blastula « die »Gastrula « und dieser die »Nenrula « folgt, so haben wir nie und nimmer $\rightarrow$ causale Einsicht gewonnen; uns fehlen dazu die analysirten Regeln des Geschehens. Wir haben nicht viel mehr als eine Geschehensfolge in der Zeit gewonnen, wobei wir gern zugeben wollen, dass Kenntnis dieser Geschehensfolge Vorbedingung causaler Einsicht ist, und wobei wir HerTwig sogar das Zugeständnis zu machen geneigt sind, dass uns bloße Beobachtung hier den Anhaltepunkt dafür geben kann, wo wir wirklich analysirte ursächliche Verhältnisse zu suchen haben. Deun dass in der »Blastula " die Ur-

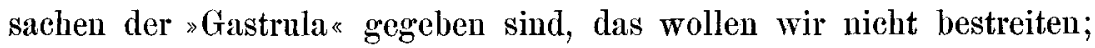
was wir leugnen ist nur, dass wir mit der durch Bcobachtung gewonnenen Kemntnis des temporalen Verhältnisses »Blastula «-»Gastrula * zugleich ein im KanT-Scropenhauer'sehen Simne causales Verhältnis eruirt hätten.

Zur Ermittelung wahrer Causalität im Morphologischen vielmehr, zur Erledigung dieser Aufgabe, welche klar formulirt und mit größter Beharrlichkeit verfolgt zu haben für immer, mag man üluer seine speciellen Leistungen und Theorien denken wie man will, ein hervor- 
ragendes Verdienst von Roux und seinen wenigen Vorgängern?(HIs etc.) bleiben wird, dazu bedürfen wir des Experiments. Wir bedürfen dazu des Versuchs, weil die Erscheinungen der Entwickelung der Organismen, welche die Beobachtung uns lehrt, ebenso wie die Veränderungen in der anorganischen Natur, so vielfach kombinirt und verwoben sind, dass auch hier nur Isolirung, Variirung und bewusste Kombination von Vorgängen - welche eben das Wesen des Experiments ausmachen - uns darïber belehren können, welche Veränderungen zu einander in unabänderlichen Beziehungen stehen und nach welehen Regeln das geschieht. Freilich ist es zu dieser Einsicht in die komplicirte Verwobenheit der ontogenetischen Erscheinungen nothwendig, sich klar zu machen, dass sich die Entwickelung einer organischen Form fur die vertiefte und analysirte Erkenntnis nicht aus "Stadien «, sondern aus relativ und vorläufig wenigstens einfachen elementaren Processen, für die ich vor einigen Jahren den Ausdruck »morphogene Elementarprocesse einführte, zusammensetzt. Von diesen Processen kann der eine noch im Verlaufe sein, während der andere bereits beendet und ein dritter eben erst inscenirt ist und so fort; obwohl in gegenseitiger Auslösungsabhängigkeit stehend, sind diese Vorgänge doch in ihrem Verlaufe, wie z. B. für die Ausbildung des Ektoderms der Echiniden von mir bewiesen wurde, in hohem Maße unabhängig von einander: eben zu erweisen, was hier von einander abhängt und was nicht, das ist die uns gestellte komplicirte Aufgabe, eine Aufgabe, die so verwickelt ist, dass wir zunächst schon froh sein müssen, wenigstens nur rein lokale Abhängigkeiten dieser Art zu ermitteln, und dass selbst zu dieser bloßen Vorarbeit für »cansale* Erkenntnis, also für eine Elkenntnis der allgemeinen » Regeln « des Geschehens ${ }^{1}$ ), bereits das Experiment einzusetzen hat.

Stiitzen wir den allgemein gewonnenen Standpunkt nunmehr im Einzelnen; vergleichen wir in besonderen Fällen, was Beobachtung und was Versuch leisten können oder geleistet haben, auf dass der

1) Trotz der hier zugegebenen Möglichkeit, dass man die allgemeine Gesetzlichkeit des gestaltlichen Geschehens, sei sie nun chemisch-physikalisch oder vitalistisch, einst ermitteln künne, bleiben natürlich die sspecifischen Lebensformen " in ihrer typischen Kombination ge gebene hinzunehmende Data, wie ich oft ausführte. Haben wir doch auch die allgemeine Gesetzlichkeit des Geschehens im Anorganischen, also z. B. des thermischen, des elektrischen Geschehens ermitteln können, obwohl uns die specifischen anorganischen Stoffe mit ihren thermischen und elektrischen Konstanten gegebene Data bleiben. 
Rangunterschied dieser Forschungsmittel um so dentlicher hervortrete. Obwohl wir unser Augenmerk besonders auf die entwickelungsphysiologische Forschung richten werden, sollen doch auch andere Gebiete der Biologie kurz in den Kreis der Betrachtung gezogen sein.

Um an eben Gesagtes anzuknïpfen, so konnte z. B. nur der Versuch die Unabhängigkeit der Mundbildung bei Echiniden von der Anwesenheit des Darmes darthun, und ans bloßer Beobachtung hätte man hier wegen des »normalen "Zusammenpassens von Mund und Darm wohl »schließen k können, dass etwa ein vom Darm ausgehender Berührungsreiz im Ektoderm die Mundbildung auslöse.

Um uns sodann einem gerade jetzt viel diskutirten Thema zuzuwenden, so haben die Versuche über die prospektive Potenz der Blastomeren gezeigt, dass im Gebiet der Entwickelungsphysiologie nicht nur zur Ermittelung wahrer Causalität, sondern, wie gesagt, sogar schon zur Ermittelung bloß funktioneller Abhängigkeitsverhältnisse, ohne Kenntnis der »Regel «, nach welcher Abhängigkeit statt hat, das Experiment nicht als Hilfsmittel zu entbehren ist. Aus reiner Beobachtung hätte man auf unbedingte $»$ Specifikation der Furchungszellen* schließen müssen, manche Forscher (zur Strassen, Jennings) haben das in gewisser Weise ja noch nenerdings, wie ich denke mit Unrecht, gethan. Der Versuch lehrte hier das Falsche dieser Lehre als allgemeiner Doktrin, er lehrte zugleich eine gewisse beschränkte Beziehung der plasmatischen Eiorganisation zur späteren Organbildung kennen, damit allerdings, wie gesagt, zunächst noch weniger ein eausales, als vielmehr ein bloß lokales Verhältnis ermittelnd.

Bezuiglich der Entwickelung von Nerven und Muskeln beruhigt sich der deskriptive Forscher dabei, dass diese beiden eben so entstehen, dass sie immer zusammen passen; will er etwas Besonderes sagen, so sagt er wohl, das sei »Vererbung «. Wenn nun Herbst $\left.{ }^{1}\right)$ glaubt, hier einen Fall von *Tropismus" vermuthen zu dürfen, das heißt einen Fall der allgemein bekannten Erscheinung, dass wachsende Organismen oder Organismentheile auf eine "Reizquelle "zuwachsen, so ist mit dieser Vermuthung einer scausalen " Auffassung die Hand gewiesen. Denn wenn durch den Versuch oder das Studium von Missbildungen diese Vermuthung einmal zur Sicherheit werden würde, so wird es wohl von Keinem geleugnet werden, dass man hier mehr gewonnen hätte, als durch eine auch noch so genaue Schilderung einer sich bietenden reinen Beobachtung.

1) Biolog. Centralbl. XIV. pag. $800 \mathrm{ff}$. 
Für die Mesenchymzellen von Echinus wies ich 1) eine solche Bewegung auf eine Reizquelle (hier eine $»$ Taxis «) durch Versuch nach: ganz unserer Definition des Experiments entsprechend variirte ich hier bewusst ein Element der zu studirenden Vorgänge, erst durch diese bewusste Isolirung war ein cansaler Schluss möglich. Wo ist der Forscher, weleher leugnen möchte, dass meine Versuche mehr lehren als die bloße Schilderung: "die Mesenchymzellen begeben sich an bestimmte Stellen des Ektoderms«. Nach welcher Regel sie sich bewegen, das eben gab ich an, womit nicht gesagt sein soll, dass meine Versuche etwa etwas endgültig Definitives geleistet hätten; aber etwas provisorisch Definitives haben sie geleistet, ebenso wie die LoEn'schen Versuche über Heliotaxis.

Hertwig meint, im Gebiet der Lehre von der Circulation sei es gezeigt worden, dass reine Beobachtung sogar mehr leisten könne als der Versuch; die einfache Beobachtung einer Froschlarve unter dem Mikroskop belehre über die Richtung der Blutbewegung besser als die komplicirten Versuche früherer Autoren. Ls ist aber zu erwägen, dass »causales "Verständnis hier iberhaupt nicht in Frage kommt, vielmehr nur die Konstatirung einer unanalysirten Thatsächlichkeit. Überhaupt ist es ja klar, dass vor jedem Versuch das Versuchsobjekt hinreichend deskriptir bekannt sein muss und wir wollen nichts weniger, als hier die große Bedeutung guter Beobachtung als Vorarbeit für causale Forschung herabsetzen. - $0 b$ andererseits für ein causales Verständnis der Blutbewegung reine Beobachtung ausreichen möchte, für eine Ermittelung der Regeln, der Naturgesetze, nach denen sie verläuft, das dürfte wohl HerTwig selbst bezweifeln. -

Oft kann eine bloß beobachtete, rein temporale oder lokale Funktionalabhängigkeit allerdings zugleich cansale Erkenntnis erwirken, nämlich dann, wenn sich das Beobachtete im Einzelnen mit Experimentalergebnissen deckt, und hinreichender Grund vorliegt, in der Beobachtung und im Experiment gleiche Umstände zu vermuthen. So sind die astronomischen Gesetze allerdings, wie HerTwig sagt, durch bloße Beobachtung gewonnen, aber er vergisst himzuzufügen, dass diese Beobachtung mit den Ergebnissen der Experimentalmechanik mathematische Identificirung gestattete. Darauf allein beruht die Sicherheit des Schlusses hier. - Bertuold und seine Nachfolger haben die Kenntnis des Antheils der Oberflächenspannung

1) Dieses Archiv. III. 3. 
am organischen Gestalten ebenfalls auf Grund solcher Analogie mit anders gewonnenen Experimentalergebnissen gewonnen. -

Die Lehre von der Befruchtung ist nach HeRTwig besonders geeignet, den Werth bloßer Beobachtung für Ergebnisse allgemeinster Bedeutnng darzuthun: dass ein Samenfaden ins Ei eindringt, dass sein Kopf sich zum Kern gestaltet etc. etc. hat Alles reine oder doch vergleichende Beobachtung gelehrt. Die Sachlage zngegeben und alle Achtung vor den Männern, die solches ermittelten: aber war hier nicht doch eine gewisse experimentelle Komponente in der Methode, indem man doch "künstliche" Befruchtung ausgefiuhrt hatte?

Oder, als Kontrast, wemn man das nicht zugeben wollte: hat man in der Befruchtungsthatsache denn mehr als eine sehr allgemeine "Thatsache", hat man überhaupt in ihr eine causale Aufhellung? Nach welcher Regel verläuft das Geschehen? Man könnte wohl nur sagen: nach der »Befruchtungsregel . Solche Erfindung von "Naturgesetzen « für einen einzigen Fall von Abhängigkeitsart verwirft aber HerTwik selbst mit Recht.

Sobald man ein wenig ins Detail der Befruchtungsvorgänge eintritt, zeigt es sich deutlich, dass man schon der komplexen Natur des hier waltenden Abhängigkeitsverhältnisses wegen durch die Beobachtung nur eine sehr allgemeine Thatsächlichkeit ermittelte.

Was bedingt die Strahlung und weiter die Spindelbildung im hefruchteten Ei? so fragte man sich; warum theilt sich das unbefruchtete Ei nicht? etc.

Hier bei Beginn analytischer und wahrhaft cansaler Betrachtung zeigte es sich nun gleich, dass man durch das Experiment allein weiter kam. So konnte aus bloBer Beobachtung des Normalen wohl vermuthet, aber nicht bewiesen werden, dass rom Centrosom Strahlung und Spindelbildung ausging: Boveri's Beobachtnng der "partiellen Befruchtung (eines "Naturexperiments — - Roux) und die bekannten Zellenversuche der Gebriuder Hentwig bewiesen aber diese Vermuthung; anch hier also war schon zu bloBer Konstatirung des analysirten Thatsächlichen der Versuch nöthig, gerade wie bei den Versuchen ïber die Prospektivität der Blastomeren: auch hier brauchte man schon ihn bei bloßer analytischer Vorarbeit zu causaler Forschung. - -

Wir halten also dafiir, dass für die Zwecke einer causalen Biologie und speciell Morphologie das Experiment das einzige universelle technische Hilfsmittel ist; Beobachtung muss ihm vorhergegangen sein, denn wer Versuche anstellt, muss sein Versuchsobjekt kennen. Das 
Experiment ist das unschätzbare Hilfsmittel der Entwickelungsphysiologie desshalb, weil uns die Entwickelungsphänomene noch mehr als die Phänomene in der anorganischen Natur in einer anßerordentlich komplicirten Verbindung und Verflochtenheit elementarer oder vorläufig elementarer Größen entgegentreten: wir miissen die Elemente bewusst variiren, isoliren und kombiniren können, um über ihren Causalzusammenhang Kenntnis zu erhalten, ja schon zu bloßer analytischer Vorbereitung zu solcher zukünftigen Kenntnis kann, wie wir sahen, der Versuch unentbehrlich sein.

In Hinsicht der Bedentung des Experiments kann ich mich also nicht mit den Erörterungen in HerTwig's nener Schrift einverstanden erklären, so sehr ich deren iibrigem Inhalte beistimme; er würdigte, so denke ich, das Experiment nicht genïgend, d. h. nicht höher als die bloße Beobachtung, weil er den Begriff des Causalen zwar richtig definirte, aber nicht sachgemä $\beta$ anwandte1). -

Neben der klaren Verfolgung der Entwickelungsphysiologie als einer selbständigen Disciplin schätze ich eben dieses als großes Verdienst Roux's, dass er zuerst, neben PfLüGer, bewusstermaßen den Versuch in der thierischen Morphologie anwandte und in systematischer Weise mit Hilfe desselben vordrang. Wenn ich leider die Mehrzahl der Roux'schen Schlussfolgerungen und Theorien fur hinfällig halten muss, so kann das an meiner Wuirdigung dieser seiner Verdienste nichts ändern.

Neapel, den 1. Februar 1897.

1) Es soll hier am Schlusse besonders betont sein, dass HERTwiG sich dagegen verwahrt, das Experiment gering zu achten; er zählt vielmehr eine große Zahl von ihm sals glänzende Entdeckungen geschätzter « Experimentaluntersuchungen auf und hat sich ja bekanntlich, wie wir Eingangs bereits betonten, selbst wiederholt experimentell bethätigt; nur als ein im Range höheres Forschungsmittel im Vergleich zur Beobachtung will er den Versuch nicht gelten lassen. Sollte er nicht vielleicht uns beigestimmt haben, wenn er eine A n a lyse der von ihm "geschätzten « anf pag. 79 und 80 namhaft gemachten Experimentalresultate versucht hätte? Wenn er sich gefragt hätte, warum er gerade sie »schätzt\& und ähnliche selbst ausführte? Leider hat er das unterlassen; er hätte sonst, dünkt mich, erkennen müssen, dass es sich gerade bei diesen Resultaten um Ermittelungen handelt, die nur mit Hilfe des Versuchs überhanpt zu gewinnen waren, weil - sie im KaNT-SCHOPENHaUER'schen Sinne cansale oder doch auf Causalitätserkenntnis hinzielende Ermittelungen sind. Durch solche Analyse hätte er dann wohl auch selbst eingesehen, dass er, wie oben bemerkt, den Causalbegriff zwar auf pag. 41 und 50 mit SCHopenHaUer's Worten zutreffend definirt, aber auf pag. 67 unzutreffend anwendet. - 